\title{
Claude Simon et Barcelone. Derechef
}

\author{
Manuel A. Tost Planet
}

\section{OpenEdition \\ Journals}

Édition électronique

URL : http://journals.openedition.org/ccs/642

DOI : $10.4000 /$ ccs. 642

ISSN : 2558-782X

\section{Éditeur :}

Presses universitaires de Rennes, Association des lecteurs de Claude Simon

\section{Édition imprimée}

Date de publication : 31 décembre 2009

Pagination : 101-117

ISBN : 9782354120580

ISSN : 1774-9425

\section{Référence électronique}

Manuel A. Tost Planet, « Claude Simon et Barcelone. Derechef », Cahiers Claude Simon [En ligne], 5 | 2009, mis en ligne le 21 septembre 2017, consulté le 21 avril 2019. URL : http:// journals.openedition.org/ccs/642; DOI : 10.4000/ccs.642 


\section{Claude Simon et Barcelone. Derechef}

La « Matière d'Espagne » (ainsi qu'on a pu la désigner à l'instar de celle de Bretagne, du moins pour la rime), devrait, en rigueur, être plutôt dite " catalane », car, quand Claude Simon parle de l'Espagne dans ses romans, c'est presque toujours en référence à Barcelone, même si, parfois, il ne l'a pas nommément citée. Mais Chklovski, le formaliste russe, ne signalait-il pas déjà, à propos de Tolstoï, que désigner un objet (au sens large du terme) sans le nommer, c'est, précisément, un bon moyen de le singulariser, en libérant le lecteur des automatismes perceptifs.'

Barcelone - et malgré qu'en aient eu à l'époque certains critiques maintenant un peu oubliés - joue donc, selon nous, un rôle important dans la construction du discours romanesque simonien. Elle y est littéralement présente et identifiable pour peu qu'on veuille bien aller dans le détail. Nous l'avons toujours souligné, en nous fondant à la fois sur la lecture des œuvres et l'observation de l'iconographie de l'époque ou de ce qu'il en reste ; conforté, qui plus est, 
par certaines précisions contenues dans des lettres de notre auteur.

Jusqu'à présent, cependant, nous n'avions pas voulu rendre publiques ces aimables lettres que le romancier avait bien voulu nous adresser, après avoir pris connaissance des écrits que nous lui avions soumis. La publication du volume des Euvres de Claude Simon dans la Pléiade par Alastair B. Duncan, avec des renvois somme toute assez nombreux, à l'endroit du Palace, que le critique britannique a bien voulu faire à notre livre ${ }^{2}$, nous incite à fournir maintenant des pistes concrètes sur ce que nous y avons avancé à propos de ce que l'on peut appeler l'insertion du réel dans le discours romanesque.

Cette correspondance, qui comprend sept lettres et sept cartes ${ }^{3}$ de longueur variable, s'échelonne de fin 1982 au début de 1993, une dizaine d'années, donc, mais de manière irrégulière, surtout à partir de 1985, comme il est facile de le comprendre. Elle est presque toute manuscrite, à part une des lettres écrite à la machine dans la première partie, d'où nous avons tiré notre premier document. Nous ne faisons état ici que d'extraits (que nous transcrivons pour en faciliter la lecture), dans la mesure où nous pouvons les accompagner, lorsque cela s'y prête, d'éléments iconographiques auxquels on peut associer des passages littéraux d'œuvres de Claude Simon. Ces références textuelles sont très nombreuses, que l'on pourrait mettre en regard de repères iconographiques.

Ces extraits de lettres de Claude Simon peuvent être disposés en quatre séries de documents correspondant à quatre motifs descriptifs que l'on retrouve dans plusieurs œuvres, mais qui ont pour référent principal Le Palace

a) Le premier concerne les portraits de personnages qu'ailleurs nous avons appelés collectifs dans la mesure où ils sont présentés presque toujours en groupes compacts : les « miliciens » anarchistes d'un côté, les «politiciens », de l'autre. Ensemble, ils font partie de la curieuse galerie de portraits de ce roman du souvenir ou du " vécu ». Différents, mais toujours présents, les « héros populaires », personnifiés dans des "espèces d'ex-votos » font partie, eux, du paysage

\footnotetext{
${ }^{2}$ Manuel A. Tost : Claude Simon. Novelas « españolas » de la guerray la revoluciôn. Barcelona: Peninsula, col Nexos, 1989, 208 p. ISBN: 978-84-297-2947-4. Voir la notice d'Alastair Duncan sur Le Palace, $E$, p. $1353,1355-1356$ et 1358.

${ }^{3}$ En fait, ces dernières sont surtout des cartes de vœux, et même si elles contiennent parfois aussi des commentaires divers, nous n'en tiendrons pas compte ici.
} 
urbain des Ramblas. (Extraits 1 à 3.)

b) Ensuite, trois extraits $(4,5$ et 6$)$ se rapportent à un personnage mythique, qui fait l'objet d'un chapitre entier dans Le Palace, «Le récit de l'homme-fusil», mais qui déborde amplement ce cadre. En effet, ce personnage, que nous avons pu identifier plus précisément, a vécu une série d'aventures rocambolesques dans de curieux concours de circonstances littéraires ou non.

c) L'extrait suivant est associé à un événement qui concerne un autre chapitre du Palace et lui donne même un intertitre, «Les funérailles de Patrocle ». En l'occurrence, il s'agit de l'enterrement d'un personnage que l'on a également pu identifier, sur les instances de Claude Simon.

d) Enfin, dans les deux derniers extraits, on ne se réfère plus à des personnages mais au contexte et à cette « fascination » que la $\mathrm{Ca}$ talogne Sud a toujours exercée sur l'auteur. À cet endroit, on aurait pu multiplier les correspondances iconiques et textuelles, mais nous laissons cette étude particulièrement intéressante, dans cette même ligne de l'insertion du réel dans le discours romanesque, pour une autre occasion... 


\section{Les « miliciens" (Paris, le 25 décembre 1982)}

I1 n'est pas

nécessaire d'avoir lu Marx, je crois, pour voir que le monde et nous mêmes sommes faits de constantes contradictions. Hi anges (comme le voulait Malraux) ni démons, mais quelque chose de bien plus émouvant, c'est-à-dire des hommes : c'est ainsi que j'ai vu les miliciens à Barcelone en 36, et il me semble encore qu'il n'y a ni condescendance ni dérision (combien de fois d'ailleurs ai-je dit dans des interviews que ce dernier sentiment m'était étranger, sinon odieux, qu' il s'agisse de miliciens ou d'un officier tué en brandissant son sabre;...) à constater ce que chacun pouvait voir alors pour peu qu'il ouvrit les yeux : à savoir qu'étant donné l'état d'affreuse misère, d'oppression et d'analphabétisme où se trouvait alors l'Espagne, il s'agissait beaucoup plus d'une "jacquerie", d' une révolte sauvage (oh combien justifiée mais, il n'empêche, sauvage) que d'une révolution (comparable, par exemple, à celle de 1789 en France).

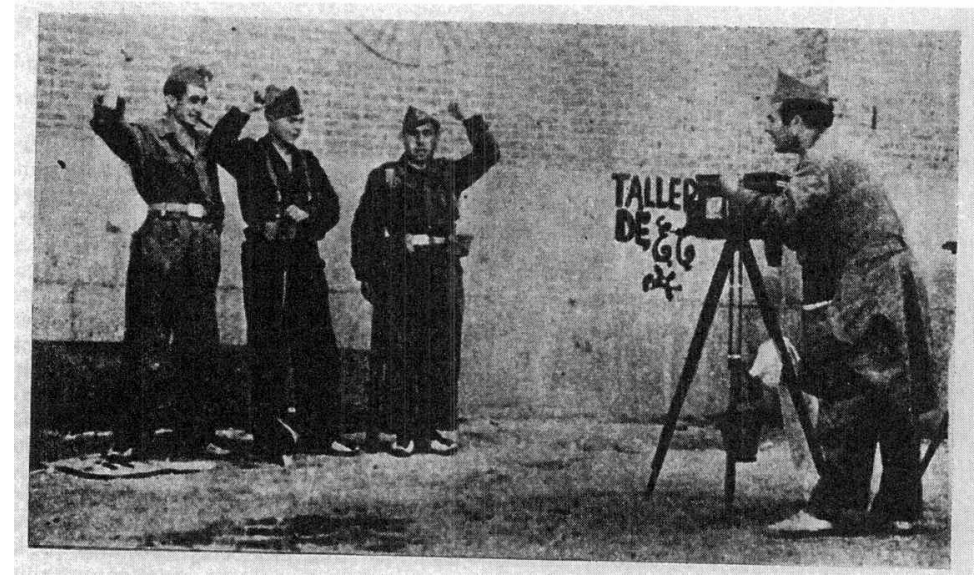

[...] décrivant un vaste cercle au-dessus de la place - avec ses photographes ambulants et leurs squameux étalages, maintenus par des pinces à linge, de photos grisâtres où posent farauds et solennels des types à têtes de berger ou de paysan empreints de cette dignité un peu compassée, un peu arrogante et un peu intimidée que confère à l'homme le fait de s'appuyer sur une arme $-[\ldots]$

Le Palace, p. $228 / 548^{4}$.

${ }^{4}$ Éditions de Minuit/Pléiade. 


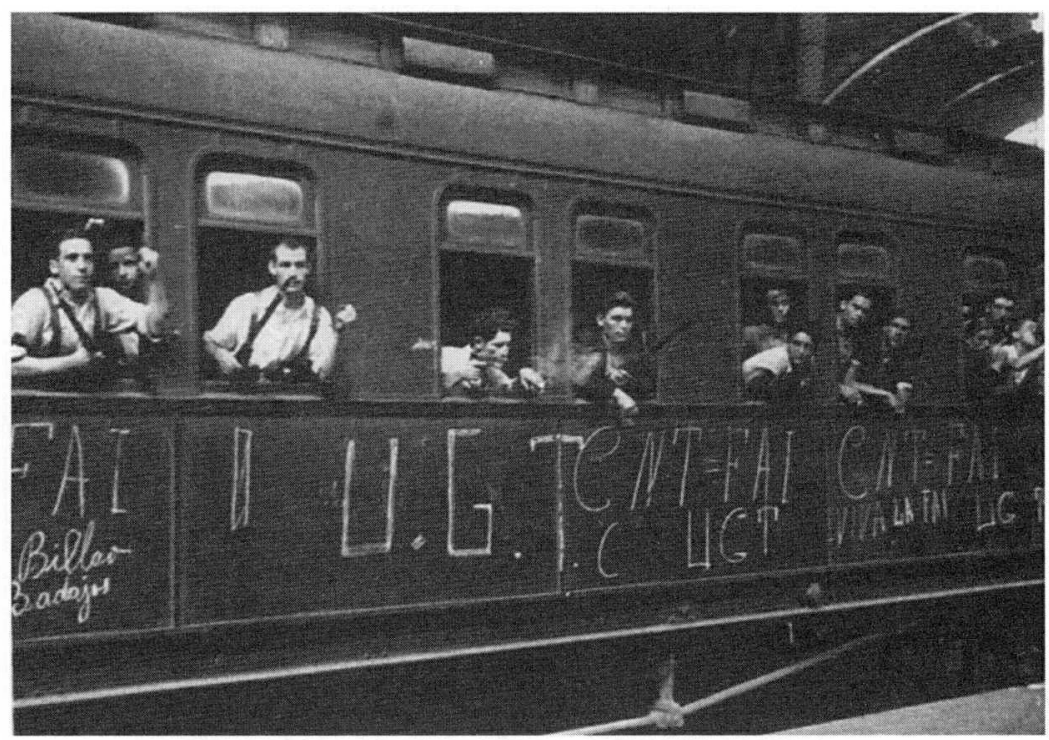

Le flanc d'un wagon, photographié légèrement de biais, occupe toute la longueur de l'image. C'est une de ces voitures massives, de construction belge, qui circulaient sur les voies à large écartement des compagnies aux capitaux étrangers installées en Espagne au début du siècle. [...] des étroites fenêtres, donc, jaillissent les bustes de jeunes gens en manches de chemise ou en combinaisons de mécanos [...] La plupart des occupants du wagon regardent le photographe et deux exhibent ostensiblement leurs fusils qu'ils tiennent inclinés, les longs canons pointant vers le ciel à l'extérieur des fenêtres. Ils ont tous d'épaisses chevelures noires, brillantes et huileuses, rejetées en arrière, saufun, atteint par un début de calvitie qui ne lui laisse que deux touffes sur les côtés [...]. La fenêtre voisine n'est occupée que par un unique milicien dont seules la tête et les épaules sont visibles, à genoux, semble-t-il, ou accroupi sur le plancher du compartiment [...].

Les Géorgiques, pp. 259-261.

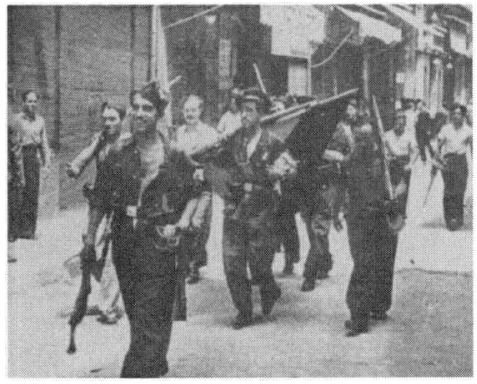

Des « types dépoitraillés brandissant leurs armes hétéroclites...» Le Palace, pp. 224/546 


\section{Les « politiciens" (Paris, le 6 janvier 1983 ; P.S.)}
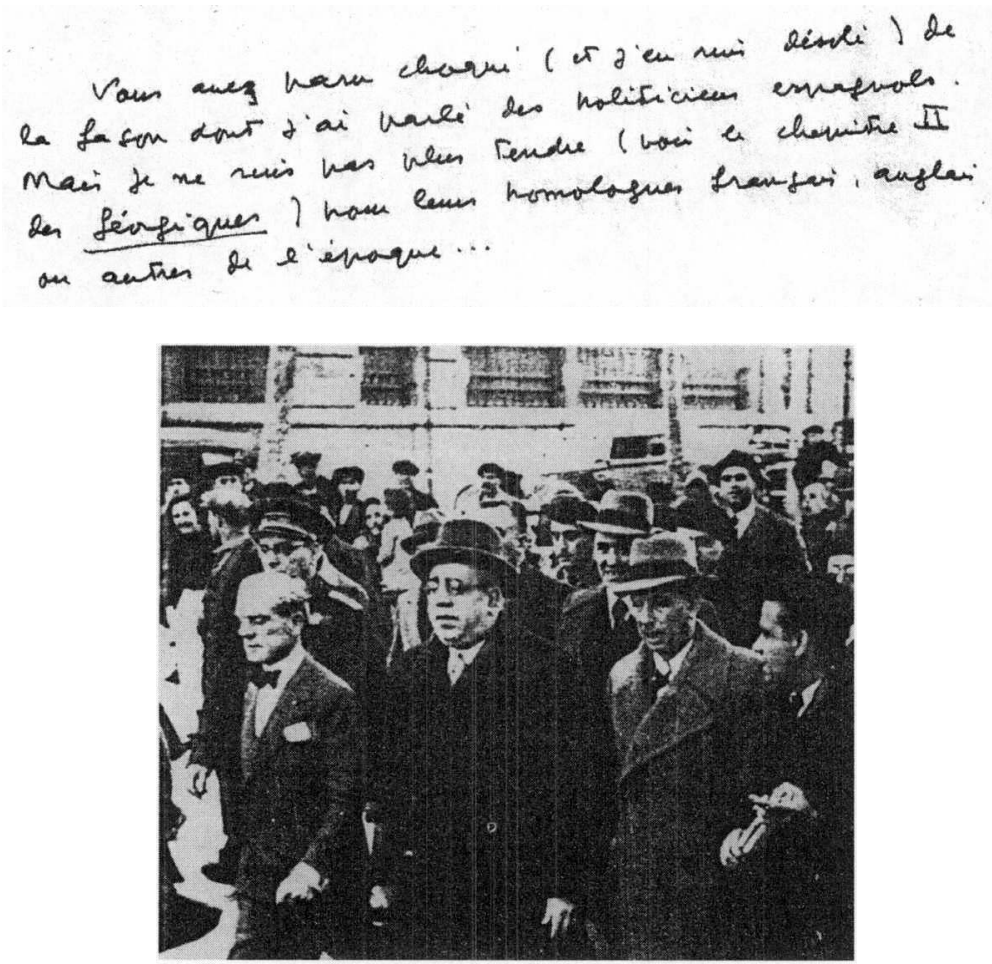

Manuel Azaña, président de la République espagnole, et Lluis Companys, président de la Generalitat de Catalogne dans une manifestation à Barcelone en 1936.

[...] la nuit des hommes armés de fusils et de revolvers qui peuvent arrêter ou tuer qui bon leur semble sans demander la permission ni même l'avis de qui que ce soit et en tout cas pas celui de la demi-douzaine de politiciens aux têtes de représentants de commerce, l'air de chantres, de moines obèses, avec leurs doubles ou triples mentons, leurs voix de basses, leurs lunettes d'écaille, leurs yeux soulignés de poches, incapables de rien d'autre que de se décerner des titres de ministres, poser complaisamment devant les photographes, palabrer à mi-voix dans les embrasures de fenêtres, se faire acclamer dans des meetings [...].

Les Géorgiques, p. 313.

[Vous avez paru choqué (et j'en suis désolé) de la façon dont j'ai parlé des politiciens espagnols. Mais je ne suis pas plus tendre (voir le chapitre II des Géorgiques) pour leurs homologues français, anglais ou autres de l'époque....] 


\section{Les « héros " et l'art populaires (Paris, le 6 janvier 1983)}

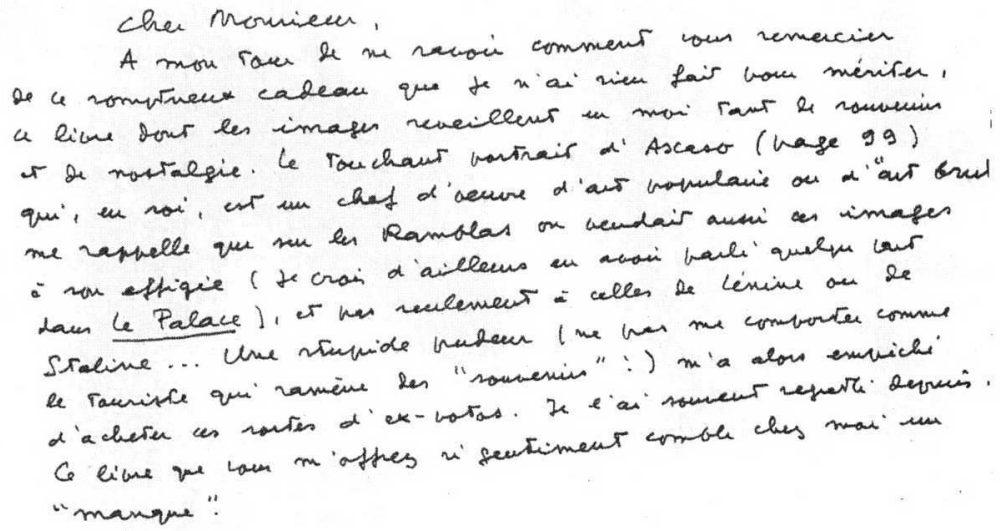

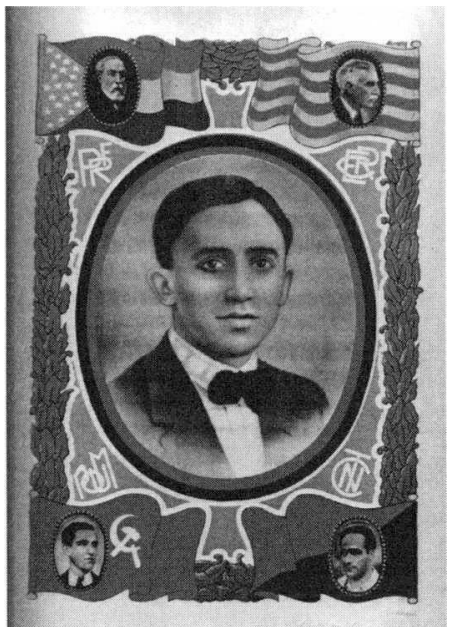

Planche en couleurs en l'honneur de Francisco Ascaso, héros anarchiste, mort au cours des premiers combats de 1936, d'un réalisme populiste très marqué, qui combine les symbologies anarchiste et catalaniste.
[...] descendant maintenant l'avenue, repassant sans les voir devant les étalages de fleurs, les panoplies guerrières, les fanions, les assortiments d'insignes et les portraits des héros morts imprimés sur foulards eux aussi entassés en piles ou accrochés par des pinces à linge à des ficelles, ondulant mollement dans le vent flasque, et qui le regardaient aussi de leurs yeux paisibles, rêveurs et légèrement surpris, non par la mort, mais sans doute par le déclic du photographe, évoquant, avec leur raie soigneusement tracée, leur veston du dimanche, leur cravate, leur expression sérieuse et puérile, moins les légendaires dynamiteurs ou braqueurs de banques, hirsutes et dépoitraillés, que (sans doute parce que le dessin du foulard a été exécuté d'après la seule photographie trouvée après coup, datant de plusieurs années auparavant) de jeunes premiers-communiants $[\ldots]$

Le Palace, pp. 213-214/539 
[Cher Monsieur,

À mon tour de ne savoir comment vous remercier de ce somptueux cadeau que je n'ai rien fait pour mériter, ce livre dont les images réveillent en moi tant de souvenirs et de nostalgie. Le touchant portrait d'Ascaso (page 99) qui, en soi, est un chef-d'œuvre d'art populaire ou d'« art brut», me rappelle que sur les Ramblas on vendait aussi ces images à son effigie (je crois d'ailleurs en avoir parlé quelque part dans le Palace), et pas seulement à celles de Lénine ou de Staline... Une stupide pudeur (ne pas me comporter comme un touriste qui ramène des " souvenirs »!) m'a alors empêché d'acheter ces sortes d'ex-votos. Je l'ai souvent regretté depuis. Ce livre que vous m'offrez si gentiment comble chez moi un "manque». ] 


\section{Karl, le communiste autrichien (Salses, le 19 août 1984)}

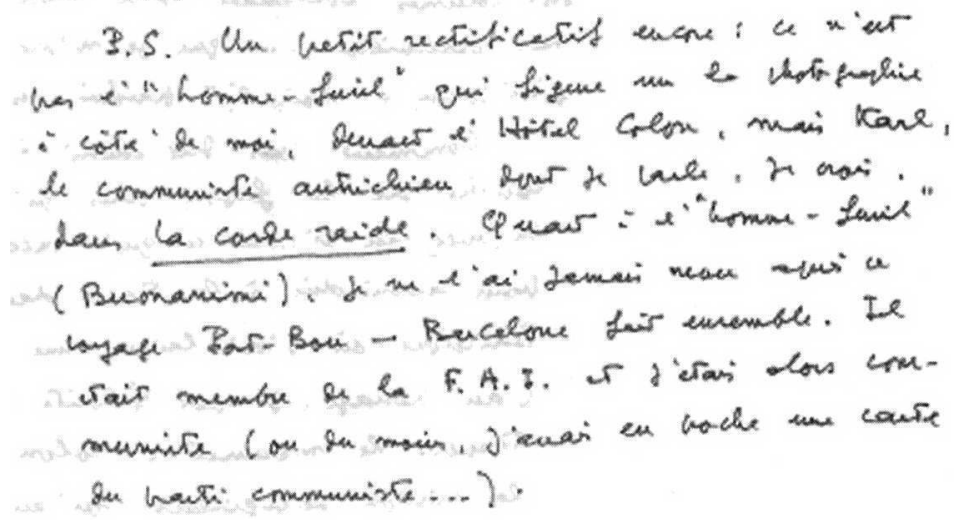

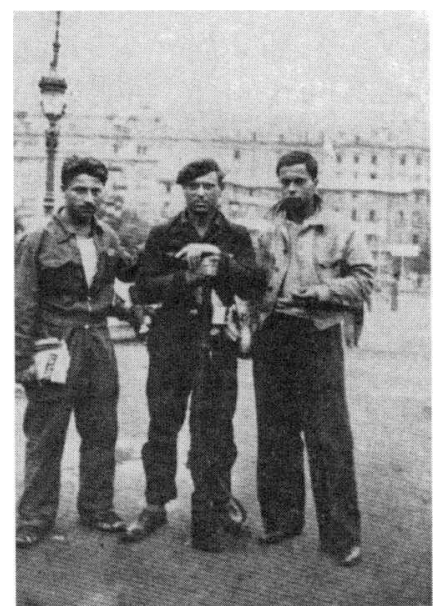

Photo bien connue fournie par l'auteur et publiée dans plusieurs ouvrages. En arrière-plan, l'hôtel Colon.

Je me rappelle la comique indignation de Carl - et pourtant je n'imagine pas qu'il eût jamais lu Goethe - un matin que les sentinelles de garde derrière la chicane de tables de café, avaient plus longuement encore que d'habitude épluché, avec leur émouvante angoisse d'illettrés, les tampons apposés sur nos " carnetes », lorsque survint une pluie soudaine et diluvienne et que tout ce qui se trouvait sur la place de Catalogne à ce moment, femmes, passants, miliciens haïs des factions rivales, courant, vestes ou jupes sur la tête, envahirent le hall de l'hôtel Colon, où traînant avec eux leurs fauteuils de rotin, s'étaient déjà réfugiés, aux premières gouttes, les soupçonneux factionnaires.

La Corde raide, pp. 44-45

[P.S. Un petit rectificatif encore : ce n'est pas l'« homme-fusil » qui figure sur la photographie à côté de moi, devant l'Hôtel Colon, mais Karl, le communiste autrichien dont je parle, je crois, dans La Corde raide. Quant à l'« homme-fusil » $\left(\right.$ Buonanimi $^{5}$, je ne l'ai jamais revu après ce voyage Port-Bou - Barcelone fait ensemble. Il était membre de la F.A.I. et j'étais alors communiste (ou du moins, j'avais en poche une carte du parti communiste...)].

\footnotetext{
${ }^{5}$ L'orthographe du nom est erronée : ce n'est pas Buonanimi, mais Bonomini.
} 


\section{Le récit de I'Homme-fusil (Paris, le 25 décembre 1982)}

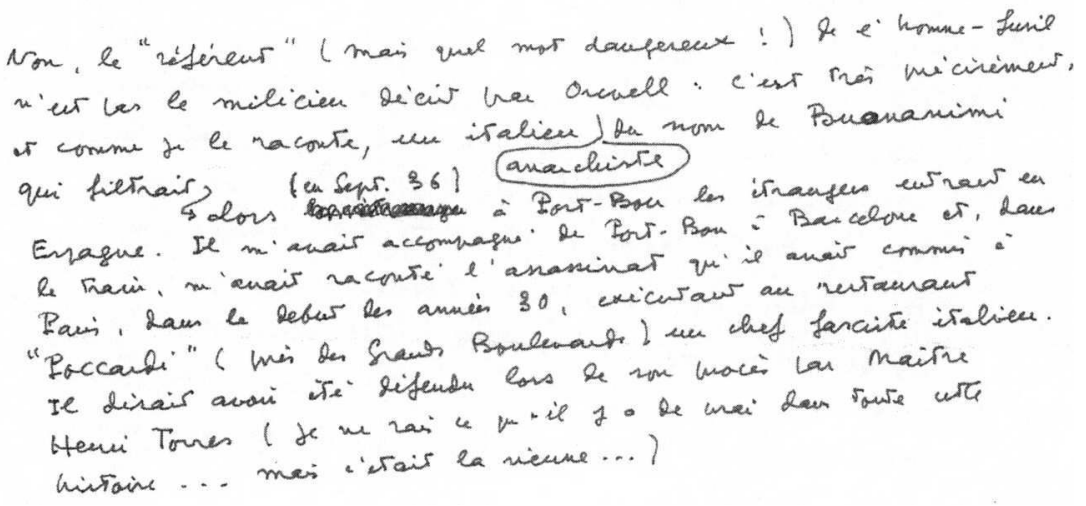

[Non, le " référent " (mais quel mot dangereux !) de l'homme-fusil n'est pas le milicien décrit par Orwell : c'est très précisément, et comme je le raconte, un Italien anarchiste du nom de Buonanimi qui filtrait alors (en septembre 36) à Port-Bou les étrangers entrant en Espagne. Il m'avait accompagné de Port-Bou à Barcelone et, dans le train, m'avait raconté l'assassinat qu'il avait commis à Paris, dans le début des années 30, exécutant au restaurant " Poccardi " (près des Grands Boulevards) un chef fasciste italien. Il disait aussi avoir été défendu lors de son procès par Maître Henri Torres (je ne sais pas ce qu'il y a de vrai dans toute cette histoire... mais c'était la sienne...)]

\section{Salses, le 19 août 1984}

\section{Ne commenaw (kelas!) que quelques mors}

Re cerrillan, i ar chenchi en wain dass wathe

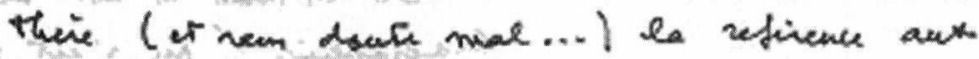

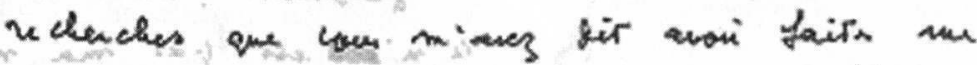

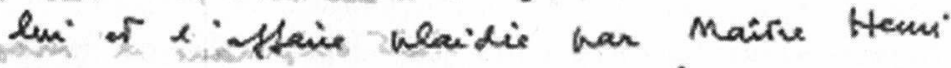

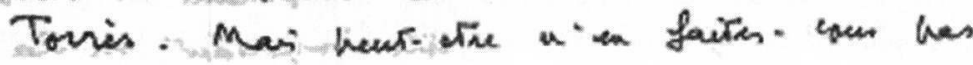
mention

[Ne comprenant (hélas !) que quelques mots de castillan, j'ai cherché en vain dans votre thèse (et sans doute mal...) la référence aux recherches que vous m'avez dit avoir faites sur lui et l'affaire plaidée par Maître Henri Torres. Mais peut-être n'en faites-vous pas mention ?...] 
Voici l'information qui faisait défaut à Claude Simon. Elle provient d'un ouvrage d'Henry Torrès, Accusés hors-série (avant-propos de Joseph Kessel), col. "L'Air du temps », NRF, Gallimard, Paris 1957. Chapitre XI, « Plaidoyers antifascistes ».

Henry Torrès écrit : "Trois procès au service de l'antifascisme marquent surtout dans ma mémoire : ceux de Pavan, meurtrier de Savorelli, de Bonomini, meurtrier de Bonservizi, de Di Modugno, meurtrier du comte Nardini. » (p. 138).

«Le procès de Bonomini, en octobre 1924, avait été celui de la dictature.

Dans une bourgade de la province de Mantoue, un adolescent est témoin des mauvais traitements infligés à son instituteur par une expédition punitive des fascistes venus de la ville. Il émigré, passant la frontière au péril de sa vie. En France, où il trouve du travail, il vit replié sur lui-même, sans amis. L'obsession le tourmente. Il envisage de rentrer en Italie pour abattre Mussolini, mais l'argent du voyage lui fait défaut.

En France, Mussolini a un ambassadeur personnel, secrétaire du fascio de Paris, directeur du Parti national fasciste, correspondant du Popolo d'Italia, directeur de l'Italie Nouvelle, M. Bonservizi. Bonomini, apprenant que celui-ci prend généralement ses repas au restaurant Noël Peters, réussit à s'y faire employer ; écrit pour sa mère une lettre contenant ce seul mot "Pardonatemi" , et se glissant, caché par un massif de fleurs, derrière la banquette où était attablé M. Bonservizi, tire sur lui deux balles à bout portant. Le malheureux s'affaisse, foudroyé. Le meurtrier déclare : "C'est ma conscience qui m'a fait agir", et, paraphrasant, sans le vouloir, Joseph de Maistre : "C'est l'idée que j'ai voulu tuer plutôt que l'homme" »(p. 139-140).

Lors du procès, Henry Torrès appela à la barre, comme témoins de la défense, plusieurs illustres personnages, Emmanuel Bourcier, Séverine, Léon Blum et Miguel de Unamuno, qui se trouvait alors réfugié en France, après son évasion de l'île de Fuerteventura 
$(\text { Canaries })^{6}$. En somme, une accumulation de coïncidences politicolittéraires.

Maintenant, on en sait un peu plus sur ce personnage qui s'était confié à l'étudiant au cours du voyage entre Port-Bou et Barcelone, et qui devint l'Homme-fusil du Palace ${ }^{7}$. Les pages web d'organisations anarchistes (sans références aux romans de Claude Simon, d'ailleurs) donnent à présent des informations sur le militant antimilitariste. Voici ce qu'en dit, de son côté, Wikipédia :

\section{Ernesto Bonomini}

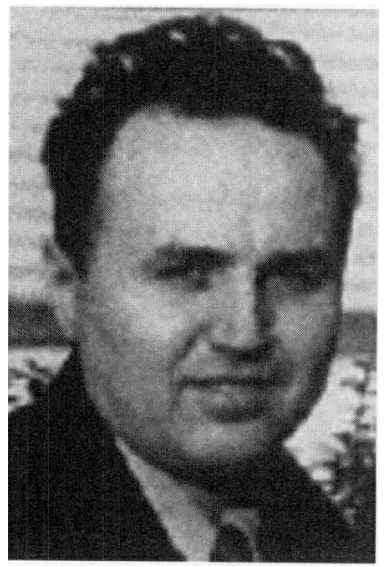

Imprégné par les idées socialistes, il devient militant antimilitariste. En 1922, persécuté par les fascistes, il quitte l'Italie pour la France. Le 20 février 1924 il tue à coups de revolver Nicola Bonservizi, responsable local du fascio (faisceau), rédacteur à Paris $\mathrm{du}$ journal fasciste L'Italie Nouvelle et correspondant du journal Popolo d'Italia. Jugé le 24 octobre 1924 devant la Cour d'Assise de la Seine, il est condamné à 8 ans de travaux forcés, peine qui sera ensuite commuée en simple emprisonnement. Libéré le 20 février 1932, il est expulsé de France au mois de juin. Il se réfugie alors en Belgique avant de venir travailler à Lille, à la Librairie Moderne. En 1935, il participe à Paris au Congrès des anarchistes italiens en exil avant de rejoindre l'Espagne où il prend une part active à la révolution et à la lutte contre le franquisme. En avril 1938, il assiste à Paris à une réunion anarchiste mais, arrêté, il est condamné à un an de prison pour avoir violé le

\footnotetext{
${ }^{6}$ Entre 1921 et 1923, Miguel de Unamuno fut vice-recteur et doyen de la Faculté de Lettres de Salamanque. Il se manifesta à plusieurs reprises contre la dictature de Primo de Rivera, dans ses écrits et ses discours, motifs pour lesquels il fut exilé à Fuerteventura. Après quelques mois d'exil il s'enfuit en France par bateau. À Paris il vécut plus d'un an (moment où il fut appelé à témoigner en faveur de Bonomini). Ensuite, il s'installa à Hendaye jusqu'à la fin de la dictature de Primo de Rivera.

${ }^{7}$ Pour d'autres détails, pas toujours concordants, sur la vie de Bonomini, voir $C E$, $1355-$ 1356.
} 
décret d'expulsion. Il est interné dans le Camp de Rieucros (Lozère) d'où il s'évade en avril 1939. Il rejoint ensuite la Belgique, puis le Canada et les États-Unis où il trouve du travail comme tapissier dans les studios d'Hollywood. Il décède à Miami le 6 juillet 1986 .

Dans l'Humanité du 5 juillet 2004, dans la rubrique "Cultures, Polars de l'été ", paraît encore un autre article, "Feux croisés ", de Robert Deleuse, qui reprend le thème et apporte de nouvelles précisions sur l'attentat.

\section{L'enterrement de «Santiago » (Paris, le 25 décembre 1982)}
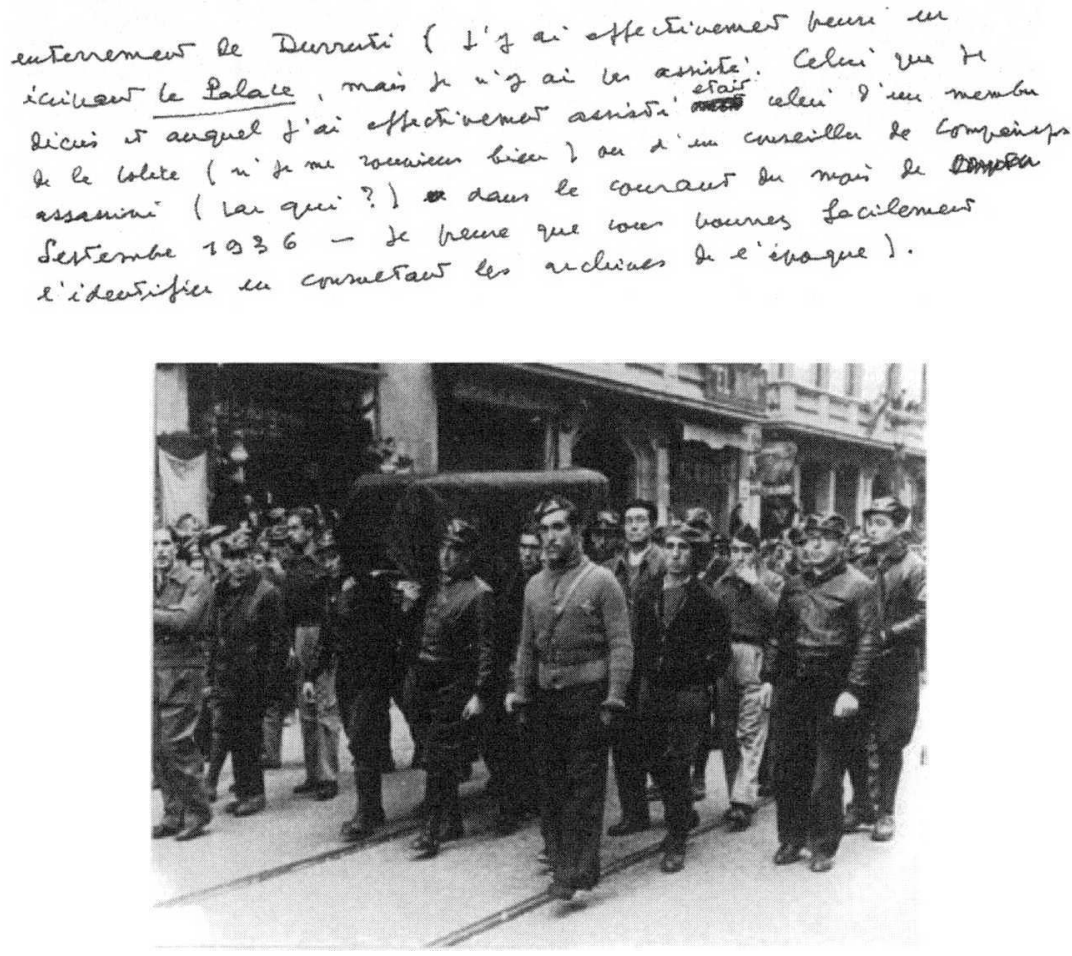

L'enterrement de Durruti

[Enterrement de Durruti (j'y ai effectivement pensé en écrivant $L e$ Palace, mais je n'y ai pas assisté. Celui que je décris et auquel j'ai effectivement assisté était celui d'un membre de la police (si je me souviens bien) ou d'un conseiller de Companys assassiné (par qui ?) dans le courant du mois de septembre 1936 - Je pense que vous pourrez facilement l'identifier en consultant les archives de l'époque.] 


\section{Entierro de un agente de policia}

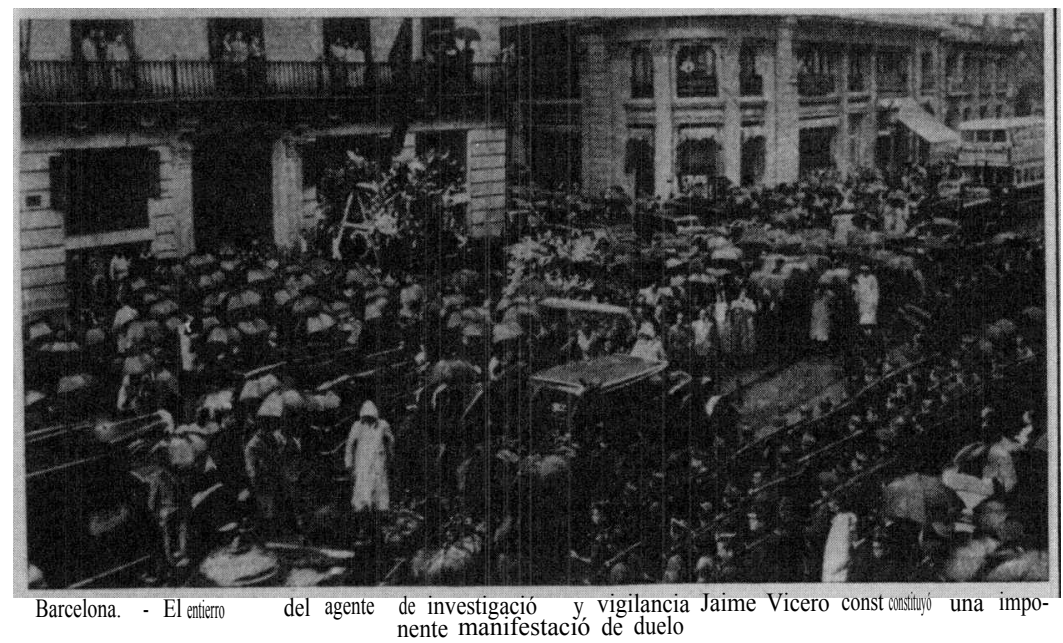

Enterrement d'un agent de la police. Légende : "Barcelone. L'enterrement de l'agent de recherche et de surveillance Jaime $e^{8}$ Vicern a constitué une imposante manifestation de deuil. " Au premier plan, le corbillard tiré par quatre chevaux.

[...] le crissement des roues maintenant très fort, à peu près, semblaitil, dans l'effroyable silence, comme un roulement de tonnerre, tandis que le corbillard passait au-dessous du balcon les quatre chevaux macabres, caparaçonnés, encapuchonnés, médiévaux, les seize pattes noires, graciles et élégantes crépitant sur la chaussée, puis le char lui-même sous la montagne de gerbes ensanglantées, ses plumes d'autruche tremblotantes, son mort invisible et meurtrier (« Les enterrements ! avait dit l'Américain. L'éternelle attraction dont on ne se fatigue jamais, l'éternel attrape-mouches pour vieillards accourant, emplis, débordant de, cachant mal la joie sénile, radoteuse et sarcastique qu'ils éprouvent [...]

Le Palace, pp. 116-117/480

\footnotetext{
${ }^{8}$ On se souvient que le nom (il faudrait plutôt dire le prénom) qui revient sans cesse dans Le Palace est Santiago. Or, en espagnol, Jaime et Santiago sont deux formes différentes (il y en a d'autres) du même prénom.
} 


\section{Le monument à Colon (Salses, le 19 août 1984)}

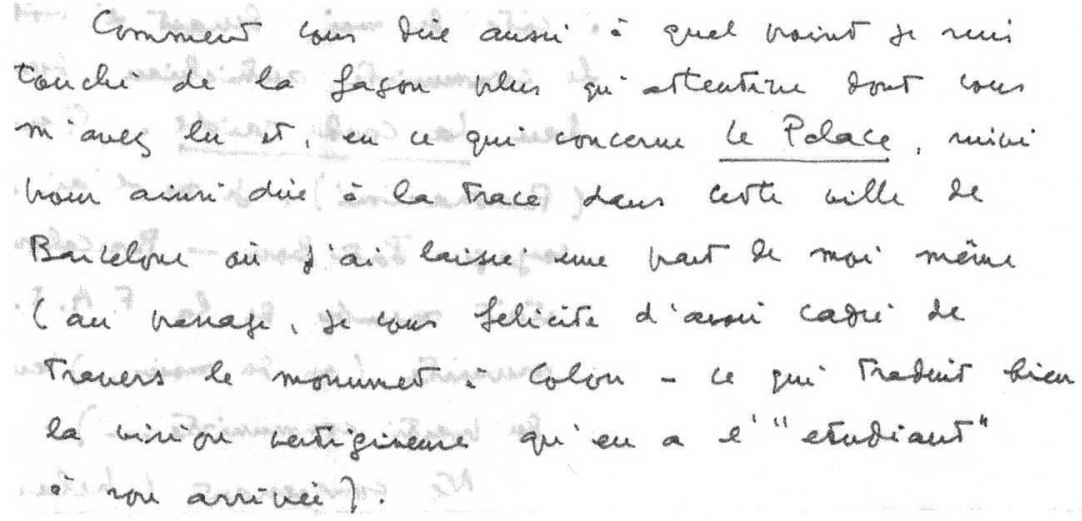

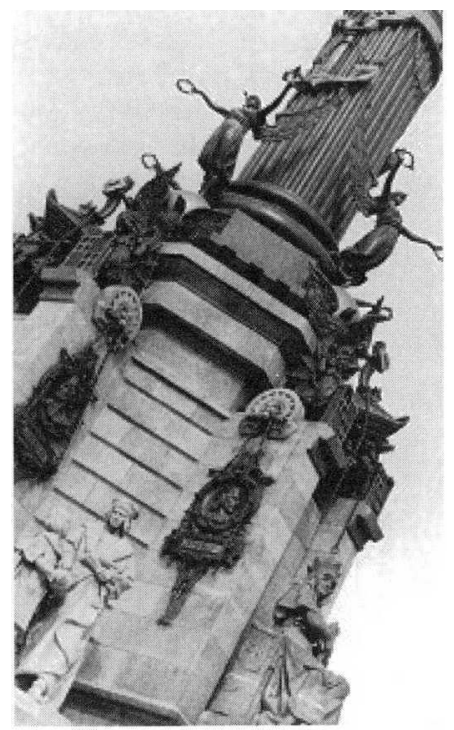

... Puis il entendit hurler les pneus et tournant brusquement la tête vers l'avant il vit quelque chose fait de pierre, de marbre et de métal entremêlés de façon compliquée et qui leur arrivait dessus à la vitesse d'une locomotive, femmes nues, tritons, rostres de navires, corniches, tout mêlé $[\ldots]$ il pouvait distinguer nettement maintenant l'impassible visage métallique d'une sirène et un entablement de pierre [...] voyant à présent s'éloigner à toute vitesse, au-dessus de son socle de figures allégoriques, de queues écailleuses, de conques et de proues, la haute colonne d'airain surmontée du globe terrestre [...].

Le Palace, pp. 81-82/459-460

[Comment vous dire aussi à quel point je suis touché de la façon plus qu'attentive dont vous m'avez lu et, en ce qui concerne Le Palace, suivi pour ainsi dire à la trace dans cette ville de Barcelone où j'ai laissé une part de moi-même (au passage, je vous félicite d'avoir cadré de travers le monument à Colon - ce qui traduit bien la vision vertigineuse qu'en a l'« étudiant » à son arrivée).] 
[...] et à ce moment il la vit, c'est-à dire ce qu'on pouvait en distinguer, de nuit et à la lumière des lampadaires, derrière le furieux défilé des troncs de platanes : une apparition, un instant, aussi furieusement emportée dans le néant qu'apparue : quelque chose d'ouvragé, paradoxal, futile : le mur aveugle et précieux aux pierres taillées en pointes de diamant, les deux niches symétriques des saints décapités, le portail encadré de colonnes torses, béant, et qui avait vomi (et, plutôt qu'un portail d'église, la gueule même de l'enfer) feu et fumée, c'est-à-dire maintenant une haute traînée noire, comme la langue de quelque monstre vorace, repu et charbonneux [...].

Le Palace, pp. 88-89/463-464

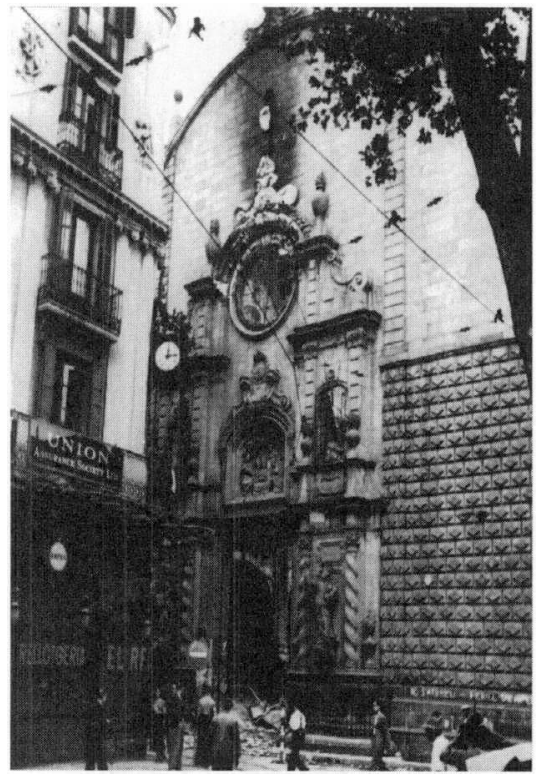

Barcelona, 19.07.1936. Incendie de l'église de N.-D. de Bethléem, sur la Rambla dels Estudis, au coin del carrer del Carme. (Institut Municipal d'Historia).

\section{9. «Une sorte de fascination" (Paris, le 6 janvier 1983)}

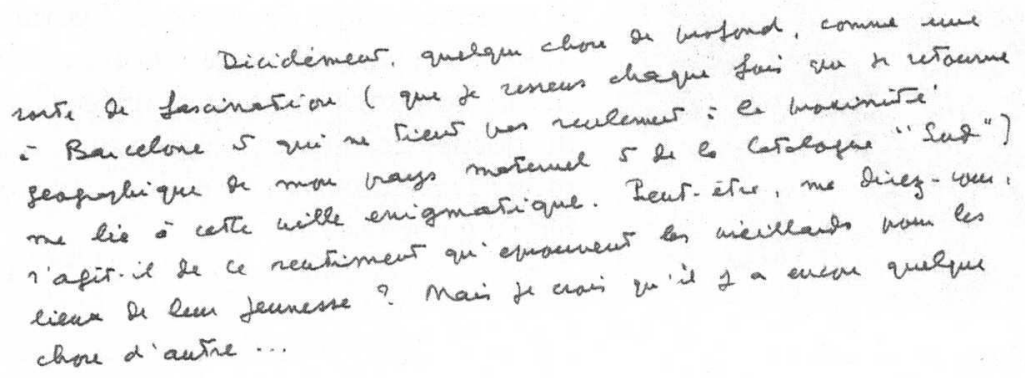

[Décidément, quelque chose de profond, comme une sorte de fascination (que je ressens chaque fois que je retourne à Barcelone et qui ne tient pas seulement à la proximité de mon pays maternel et de la Catalogne "Sud») me lie à cette ville énigmatique. Peut-être, me direz-vous, s'agit-il de ce sentiment qu'éprouvent les vieillards pour les lieux de leur jeunesse? Mais je crois qu'il y a encore quelque chose d'autre...] 


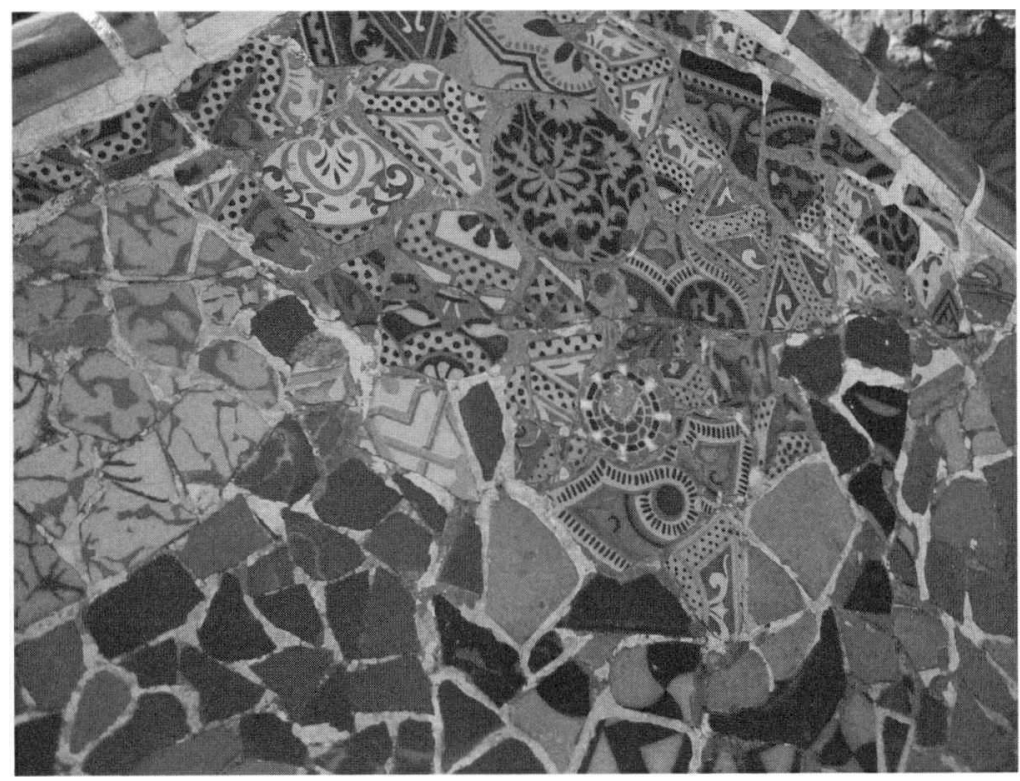

L. - Vous insistez souvent sur la fragmentation dans l'art moderne?

C.S. - Peut-être faudrait-il parler simplement de l'art tout court et non pas seulement de l'art moderne. Qu'y a-t-il par exemple de commun entre des fruits peints par Caravage ou par Cézanne, un visage de femme de Cimabue ou de Rubens, un arbre de Poussin ou de Van Gogh ? Chacun n'a retenu qu'un aspect, un « fragment » de la " réalité ». En ce qui concerne l'art contemporain, on pourrait parler de " cassures » de la forme, d'un certain éclatement. Mais continu et discontinu, fragment et totalité sont des questions terriblement complexes à analyser [...]. Tout au plus peut-on chercher à dater les premières manifestations. À ce titre, le parapet du Parque Giiell construit par Gaudi à Barcelone et dont il a décoré les surfaces courbes de fragments de carreaux de céramique, brisés et assemblés ensuite dans une longue composition dont les lois ne sont plus celles de la continuité du dessin, mais d'autres rapports, me semble le point de départ. Cette œuvre monumentale se déploie sur environ cent cinquante mètres et soyez sûr que, de Picasso à Louise Nevelson en passant par Matisse, Miró et Rauschenberg, tout le monde est venu la regarder et y apprendre.

Didier Éribon : «Fragments de Claude Simon », (entretien), Libération, 29-30 août 1981. 